\title{
Chelation of Mitochondrial Iron Prevents Seizure-Induced Mitochondrial Dysfunction and Neuronal Injury
}

\author{
Li-Ping Liang, Stuart G. Jarrett, and Manisha Patel \\ Department of Pharmaceutical Sciences, University of Colorado Denver, Aurora, Colorado 80045
}

\begin{abstract}
Chelatable iron is an important catalyst for the initiation and propagation of free radical reactions and implicated in the pathogenesis of diverse neuronal disorders. Studies in our laboratory have shown that mitochondria are the principal source of reactive oxygen species production after status epilepticus (SE). We asked whether SE modulates mitochondrial iron levels by two independent methods and whether consequent mitochondrial dysfunction and neuronal injury could be ameliorated with a cell-permeable iron chelator. Kainateinduced SE resulted in a time-dependent increase in chelatable iron in mitochondrial but not cytosolic fractions of the rat hippocampus. Systemically administered $N, N^{\prime}$-bis (2-hydroxybenzyl) ethylenediamine- $N, N^{\prime}$-diacetic acid (HBED), a synthetic iron chelator, ameliorated SE-induced changes in chelatable iron, mitochondrial oxidative stress (8-hydroxy-2' deoxyguanosine and glutathione depletion), mitochondrial DNA integrity and hippocampal cell loss. Measurement of brain HBED levels after systemic administration confirmed its penetration in hippocampal mitochondria. These results suggest a role for mitochondrial iron in the pathogenesis of SE-induced brain damage and subcellular iron chelation as a novel therapeutic approach for its management.
\end{abstract}

Key words: kainic acid; epilepsy; oxidative stress; iron chelator; glutathione; mtDNA damage

\section{Introduction}

Iron has been suggested to be one of the most abundant metals in the brain (Beard et al., 1993). Abnormal iron accumulation is a pathological feature of various neurological diseases, most notably Parkinson's disease, Alzheimer's disease, Huntington's disease and Friedreich's ataxia (Zecca et al., 2004). Although the vast majority of iron is bound to proteins, a small percentage of cellular iron termed "chelatable iron" exists "in transit" between cellular uptake and binding to iron storage proteins and is amenable to chelation (Evans and Halliwell, 1994; Petrat et al., 2002a). Chelatable iron bound to small molecules in the cellular milieu can be redox reactive and has been implicated in tissue injury (Halliwell and Gutteridge, 1990; Petrat et al., 2002a). Recent studies have shown that chelatable iron is not only present in the cytosol, but also in other cellular compartments such as nuclei, mitochondria and lysosomes (Petrat et al., 2001, 2002b; Glickstein et al., 2005). In contrast to protein bound iron, chelatable iron can facilitate oxidative damage to lipids, DNA and susceptible proteins by Fenton chemistry (Zecca et al., 2004). Consistent with the deleterious nature of iron, nutritional iron deprivation has been shown to attenuate excitotoxic neuronal injury (Shoham and Youdim, 2004).

Chelation of redox-active transitional metals has the potential to prevent oxidative stress and neuronal loss. Chelators such as clioquinol and desferrioxamine (DFO), have been shown to exert

Received June 30, 2008; revised Aug. 7, 2008; accepted Sept. 23, 2008.

This work was supported by National Institute of Neurological Disorders and Stroke Grants R01NS39587-07 and NS045748-04 (M.P.).

Correspondence should be addressed to Dr. Manisha Patel, Department of Pharmaceutical Sciences, Research Building 2, 12700 East 19th Avenue, Aurora, C0 80045. E-mail: manisha.patel@uchsc.edu.

D01:10.1523/JNEUROSCI.3016-08.2008

Copyright $\odot 2008$ Society for Neuroscience $\quad 0270-6474 / 08 / 2811550-07 \$ 15.00 / 0$ neuroprotection in models of Alzheimer's and Parkinson's disease (Alam et al., 1997; Cherny et al., 2001; Kaur et al., 2003). However, these compounds have several drawbacks with respect to specificity, toxicity and blood brain barrier (BBB) penetration. (Ben-Shachar et al., 1991; Arbiser et al., 1998; Zecca et al., 2004). $N, N^{\prime}$-bis (2-hydroxybenzyl) ethylenediamine- $N, N^{\prime}$-diacetic acid (HBED) is a synthetic lipophilic cell-permeable chelator which has been shown to eliminate twice the iron on an equimolar basis in comparison with DFO and has been evaluated in non-human primates and human volunteers (Bergeron et al., 1999, 2002). It is well absorbed from the gastrointestinal tract and retains activity as an iron chelator after oral administration in rodents. HBED has successfully completed preclinical evaluation in animals without evidence of adverse effects (Brittenham, 2003). Importantly, HBED has been shown to protect mitochondrial targets and chelate iron therein (Kalivendi et al., 2003).

High iron concentrations predominate in brain regions such as the substantia nigra and the hippocampal CA3 region (Hill and Switzer, 1984). Recent findings suggest that impairment of mitochondrial function occurs in both human and experimental epilepsy (Kudin et al., 2002; Liang and Patel, 2004b). Acute seizure activity results in increased production of reactive species, oxidative damage to cellular targets, impaired mitochondrial redox status, mitochondrial dysfunction and apoptosis (Cock, 2002; Patel, 2004; Henshall and Simon, 2005). Work from our laboratory demonstrates that mitochondria are the major cellular site of oxidative stress generation after acute SE (Liang et al., 2000; Liang and Patel, 2004b, 2006; Patel et al., 2008). Although the epileptogenic role of exogenous iron salts or blood has been recognized for decades (Willmore et al., 1978; Hammond et al., 1980), the role of intracellular iron in mediating seizure-induced brain damage remains unknown. Therefore, we determined whether 
SE results in the accumulation of mitochondrial chelatable iron, and whether chelation of iron by HBED protects against SEinduced mitochondrial dysfunction and neuronal injury.

\section{Materials and Methods}

Animals. Animal studies were performed in accordance with the $\mathrm{Na}$ tional Institute of Health Guide for the Care and Use of Laboratory Animals (NIH Publications No. 80-23). All procedures were approved by the Institute Animal Care and Use Committee (IACUC) of the University of Colorado at Denver and Health Sciences Center (UCDHSC), which is fully accredited by the American Association for the Accreditation of Laboratory Animal Care.

Kainic acid (KA) and $\mathrm{N}, \mathrm{N}^{\prime}$-bis (2-hydroxybenzyl) ethylenediamine$\mathrm{N}, \mathrm{N}^{\prime}$-diacetic acid (HBED) administration. Adult male Sprague Dawley rats ( $\sim 3$ months of age, weighing $\sim 300-350 \mathrm{~g}$ ) were injected subcutaneously (s.c.) with a single dose $(12 \mathrm{mg} / \mathrm{kg}$ ) of KA (Ocean Products International) dissolved in sterilized saline. HBED (Strem Chemicals) was injected at the dose of $75 \mu \mathrm{mol} / \mathrm{kg}$, s.c. daily three times before KA and once after KA for a total of four injections. HBED was dissolved in dimethyl sulfoxide (DMSO) and diluted with sterilized phosphate buffered saline (PBS) to achieve the desired final concentration (1\% DMSO). The animals were divided into four different groups: (1) control; (2) HBED; (3) KA; (4) KA+HBED. The severity of behavioral seizures was evaluated for $8 \mathrm{~h}$ after KA by a scale previously described (Ben-Ari et al., 1981; Baran et al., 1987; Liang et al., 2000): 0, normal; 1, immobilization, occasional "wet-dog shakes"; 2 , head nodding, unilateral forelimb clonus, frequent "wet dog shakes"; 3 , rearing, salivation, bilateral forelimb clonus; 4 , generalized limbic seizures with falling, running and salivation; 5 , continuous generalized seizures with tonic limbic extension, death. Behavioral seizure latency was calculated from the time ( $\mathrm{min}$ ) of injection of KA to the first episode of "wet dog shakes." The animals developed generalized tonic-clonic convulsions with frequent rearing and falling which was rated 3-4 on the severity of behavioral seizures. The behavioral seizures started $\sim 90-120 \mathrm{~min}$ and peaked during the third and fourth hours after injection of KA. The seizures usually persisted for $5-6 \mathrm{~h}$ and gradually ceased. The time from the beginning to cessation of generalized behavioral seizures was recorded individually as the approximate duration of status epileptics.

Isolation of subcellular hippocampal fractions. Mitochondria were isolated from pooled hippocampi of individual rats according to the method previously described (Liang and Patel, 2006). The hippocampus was homogenized with a Dounce tissue grinder in mitochondrial isolation buffer ( $70 \mathrm{~mm}$ sucrose, $210 \mathrm{~mm}$ mannitol, $5 \mathrm{~mm}$ Tris $\mathrm{HCl}, 1 \mathrm{~mm}$ EDTA; pH 7.4. with the exception of isolation buffer for measurement of chelatable iron which did not contain EDTA.) The suspensions centrifuged at $800 \times g 4^{\circ} \mathrm{C}$ for $10 \mathrm{~min}$. The supernatants were centrifuged at $13,000 \times g 4^{\circ} \mathrm{C}$ for $10 \mathrm{~min}$, pellets washed with mitochondrial isolation buffer and centrifuged at $13,000 \times g 4^{\circ} \mathrm{C}$ for 10 min to obtain mitochondrial fractions. The purity of mitochondrial fractions has been confined by immunoblot analysis of cytochrome $c$ oxidase (COX) (EC1.9.3.1) subunit IV and lactate dehydrogenase (LDH) (EC 1.1.1.27) in mitochondrial and cytosolic fractions (Liang and Patel, 2006).

Intracellular total iron quantified by colorimetric ferrozine assay. Total brain iron levels were assayed by a method previously descried (Riemer et al., 2004). Briefly, rat brains were sonicated in $50 \mathrm{~mm} \mathrm{NaOH} \mathrm{(10 \%} \mathrm{w/v).}$ Brain homogenates $(100 \mu \mathrm{l})$ placed in Eppendorf tubes were mixed with $100 \mu \mathrm{l}$ of $\mathrm{HCl}(10 \mathrm{~mm})$ and $100 \mu \mathrm{l}$ of the iron-releasing reagent which contained a freshly mixed solution of equal volumes of $1.4 \mathrm{M} \mathrm{HCl}$ and $4.5 \%(\mathrm{w} / \mathrm{v}) \mathrm{KMnO}_{4}$ in $\mathrm{H}_{2} \mathrm{O}$. The mixture was incubated for $2 \mathrm{~h}$ at $60^{\circ} \mathrm{C}$. Previous reports have shown that the $\mathrm{HCl} / \mathrm{KMnO}_{4}$ pretreatment is sufficient to release iron quantitatively from proteins, including the ironstorage protein ferritin (May and Fish, 1978). Mixtures were cooled to room temperature, $30 \mu \mathrm{l}$ of iron-detection reagent added to each tube and further incubated for $30 \mathrm{~min}$ at $37^{\circ} \mathrm{C}$. An aliquot of the solution $(300$ $\mu \mathrm{l})$ from each tube was placed into a well of a 96-well plate and the absorbance read at $550 \mathrm{~nm}$ on a UV microplate reader. The iron content of the sample was calculated by comparing its absorbance to that of a range of standard concentrations of equal volume that had been prepared
Chelatable Iron

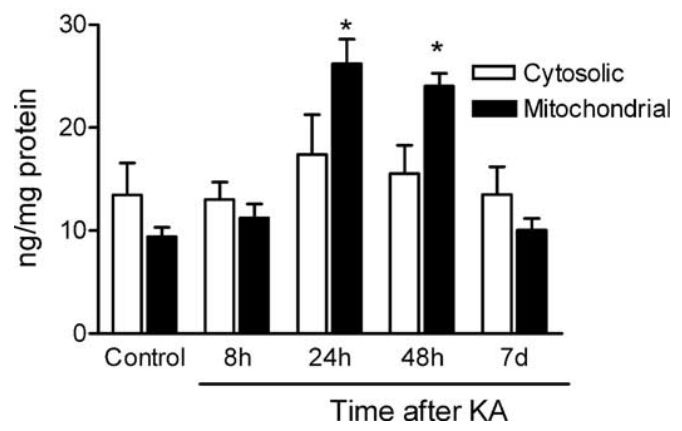

Figure 1. The levels of chelatable iron in hippocampal cytosolic and mitochondrial fractions of rats at varying time points after KA administration. Bars represent the mean $+\mathrm{SEM},{ }^{*} p<$ 0.01 vs saline control; one-way ANOVA, $n=6$ rats per group.

in a manner similar to that of the sample at concentrations of iron ranging from $12.5-200 \mathrm{ng} / 100 \mu \mathrm{l}$.

Measurement of chelatable iron by the bleomycin method. Chelatable iron was measured in subcellular fractions as previously described (Liang and Patel, 2004a) developed with minor modifications from a method described by Evans and Halliwell (Evans and Halliwell, 1994; Burkitt et al., 2001). All reagents were treated with Chelex $100 \operatorname{Resin}(0.3 \mathrm{~g} / 10 \mathrm{ml})$ to remove contaminating iron. The samples were incubated in a $1 \mathrm{ml}$ volume of reagents $(1 \mathrm{mg} / \mathrm{ml}$ calf thymus DNA, $1.5 \mathrm{U} / \mathrm{ml}$ bleomycin, $5 \mathrm{~mm}$ $\mathrm{MgCl}_{2}, 100 \mathrm{~mm}$ Tris- $\mathrm{HCl} \mathrm{pH} \mathrm{7.4,} 1 \mathrm{~mm}$ ascorbate and $50 \mu \mathrm{l} \mathrm{sample)} \mathrm{for}$ $1 \mathrm{~h}$ in a shaking water bath at $37^{\circ} \mathrm{C}$. The reaction was stopped by addition of $0.1 \mathrm{ml}$ of $60 \mathrm{~mm}$ butylated hydroxytoluene (BHT). The sample was mixed with $0.2 \mathrm{M}$ phosphoric acid, $0.11 \mathrm{~m}$ thiobarbituric acid (TBA), the chromagen extracted and absorption of the organic layer read at $532 \mathrm{~nm}$. Iron concentrations were determined in samples by using a series of standard iron concentrations $(1-100 \mathrm{ng} / \mathrm{ml})$.

Rhodamine B-[(1,10-phenanthrolin-5-yl) aminocarbonyl]benzyl ester (RPA) fluorescence analysis. Measurement of iron by RPA (Squarix Biotechnology) staining was performed by methods previously described by Petrat et al. (2002a) with minor modifications. Briefly, frozen coronal brain sections were air dried for $30 \mathrm{~min}$ at room temperature, incubated with RPA ( $3 \mu \mathrm{M}$ in phosphate buffered saline; PBS) for $30 \mathrm{~min}$ at $37^{\circ} \mathrm{C}$, followed by additional $10 \mathrm{~min}$ incubation in PBS. The sections were further dried for $30 \mathrm{~min}$ at room temperature, cleared by immersion in xylene and coverslipped with DPX (a nonaqueous nonfluorescent plastic mounting medium for histology, Fluka). The images were captured using a Nikon Optiphot-2 80i microscope equipped with epifluorescense optics. RPA fluorescence of a given area was automated and estimated with Image J (National Institutes of Health, Bethesda, MD), an open source image manipulation tool, in three sections, $100 \mu \mathrm{m}$ apart in the hippocampal CA1, CA3 and hilus subfields from both hemispheres of each animal. The average relative fluorescence density was expressed as a percentage of control values.

Measurement of HBED levels. HBED was measured by HPLC with UV detection. The whole hippocampus or mitochondrial fractions from the hippocampus were sonicated in $100 \%$ methanol. The homogenates were incubated at room temperature for $10 \mathrm{~min}$ to extract $\mathrm{HBED}$, added to an equal volume of $0.1 \mathrm{~m}$ perchloric acid and centrifuged at $13,000 \times g$ at $4^{\circ} \mathrm{C}$ for $15 \mathrm{~min}$ to precipitate protein. Aliquots $(50 \mu \mathrm{l})$ of the supernatant were injected into HPLC. HBED was analyzed with UV detector at $221 \mathrm{~nm}$ and a 3 $\mu \mathrm{m}, 150 \times 4.6 \mathrm{~mm}$ YMC Basic column (Waters Corporation). Mobile phase was composed of $25 \mathrm{~mm}$ sodium acetate, $30 \%$ acetonitrile adjusted to $\mathrm{pH} 3.0$ with trifluoroacetic acid and flow rate was set at $0.7 \mathrm{ml} / \mathrm{min}$.

HPLC measurement of 8-hydroxy-2-deoxyguanosine (8-OHdG) and 2-deoxyguanosine (2-dG). DNA isolation procedure was performed with 3 times chloroform/isoamyl alcohol extraction $(24: 1, \mathrm{v} / \mathrm{v})$ as described previously (Liang et al., 2000). DNA digestion was performed as described previously (Kasai et al., 1986). The oxidative DNA adduct, 8-OHdG was measured with HPLC equipped with electrochemical and 
UV detection following the methods described before (Shigenaga et al., 1990; Liang et al., 2000). Electrochemical detector potentials for 8-OHdG analyses were 120/230/280/420/600/ $750 \mathrm{mV}$ (vs Pd). The wavelength for UV detection of $2-\mathrm{dG}$ was set at $260 \mathrm{~nm}$. The samples were separated on a $3 \mu \mathrm{m}, 150 \times 4.6 \mathrm{~mm}$ YMC Basic column (Waters Corporation). The mobile phase was composed of $50 \mathrm{~mm}$ sodium acetate, $5 \%$ methanol; pH 5.2 and flow rate was $1.0 \mathrm{ml} / \mathrm{min}$.

Measurement of reduced and oxidized glutathione (GSH and GSSG). GSH and GSSG were measured by HPLC equipped with electrochemical detection (HPLC-EC) as described previously (Lakritz et al., 1997; Liang and Patel, 2006). Electrochemical detector potentials were 120/355/480/560/700/820 mV (vs Pd).

$m t D N A$ damage analysis. Genomic DNA was extracted using a commercially available Qiagen DNeasy Tissue kit. The quantitative polymerase chain reaction (QPCR) assay measures the average oxidative lesion frequency and works on the premise that an oxidative lesion on the DNA template will block a thermostable polymerase and result in reduced amplification of the mtDNA fragment. Therefore, only those DNA templates that do not contain polymerase blocking lesions will be amplified. The DNA damage was quantified by comparing the relative efficiency of the amplification of a $13.4 \mathrm{~kb} \mathrm{mtDNA}$ gene fragment. mtDNA lesion frequencies were calculated as the amplification of damaged (treated) samples $\left(A_{\mathrm{d}}\right)$ relative to the amplification of nondamaged small fragment controls $\left(A_{0}\right)$ resulting in the ratio $\left(A_{\mathrm{d}} / A_{0}\right)$. To determine average lesion frequency per $10 \mathrm{~kb}$, a random distribution of lesions was assumed, and the following equation was used, $\lambda=-\ln A_{\mathrm{d}} / A_{0}(0.7)$. PCR conditions used in this study were based on previously reported sequences for mtDNA primers and normalized to lesions per $10 \mathrm{~kb}$, with minor modifications (Yakes and Van Houten, 1997; Ayala-Torres et al., 2000). The primer sequences were as follows for the rat mitochondrial genome $5^{\prime}$ CCT CCC ATT CAT TAT CGC CGC CCT TGC $3^{\prime}$ and 5' GAT GGG GCC GGT AGG TCG ATA AAG GAG 3'. QPCR was performed on a DNA Engine thermal cycler. The intensity of the PCR product bands was quantified with Scion Image analysis software (Version Beta 4.0.2).

Fluoro-Jade B analysis. Rats were killed $7 \mathrm{~d}$ after KA administration and frozen sections $(15 \mu \mathrm{m})$ were cut coronally and stained with Fluoro-Jade B (Histo-Chem) as described in the literature (Hopkins et al., 2000) and instructions provided by the company. Briefly, the sections were dried at $50^{\circ} \mathrm{C}$ for $30 \mathrm{~min}$ and immersed in a solution containing $1 \%$ sodium hydroxide in $80 \%$ alcohol for $5 \mathrm{~min}$. This was followed by $2 \mathrm{~min}$ incubation in $70 \%$ alcohol and $2 \mathrm{~min}$ in distilled water. The sections were transferred to a solution of $0.06 \%$ potassium permanganate for $10 \mathrm{~min}$ and rinsed in distilled water for $2 \mathrm{~min}$. The staining solution was prepared from a $0.004 \%$ Fluoro-Jade B in $0.1 \%$ acetic acid vehicle. The solution was typically prepared within $10 \mathrm{~min}$ of use and was not reused. After incubating for $15 \mathrm{~min}$ in the staining solution, the sections were rinsed for $1 \mathrm{~min}$ in each of three distilled water washes and dried. The dry sections were cleared by immersion in xylene for at least a minute before coverslipping with DPX. Images were captured using a Nikon Optiphot-2 80i microscope equipped with epifluorescense optics. The Fluoro-Jade B positive signal of a given area was measured with Image J software as described in RPA fluorescence quantification. The average of the fluorescent relative density was expressed as percentage of the control.

Statistical analyses. For comparison among three or more treatment groups, one-way ANOVA with Tukey's post hoc test was used. $p$ values $<0.05$ were considered significant.

\section{Results}

\section{SE increases mitochondrial chelatable iron}

To determine whether SE resulted in subcellular iron accumulation, we measured chelatable iron in cytosolic and mitochondrial fractions from hippocampus of rats injected with KA. Chelatable iron was measured by the bleomycin assay
Behavioral seizure severity

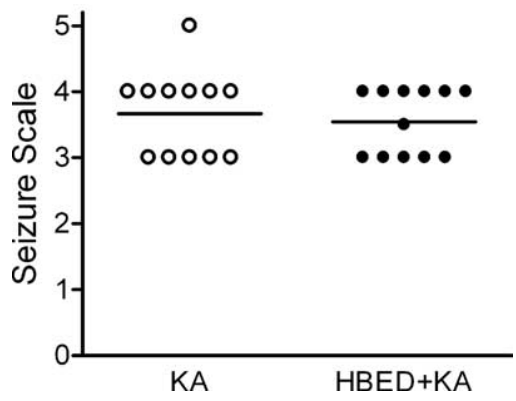

Figure 3. Behavioral seizure severity in rats after injection with KA alone or KA +HBED. $n=12$.

which has been routinely used as a sensitive and reliable means of measuring free iron in whole blood (Ando et al., 2002), plasma (Burkitt et al., 2001) and tissue samples (Sohal et al., 1999; Andreassen et al., 2001; Kirk et al., 2001). In control rats, the average concentration of chelatable iron in the cytosolic and mitochondrial fractions of the hippocampus was $\sim 15 \mu \mathrm{M}$ which is comparable to previous literature reports of chelatable iron concentrations in mitochondrial and cytosolic fractions of hepatocytes (Petrat et al., 2001, 2002b). KA administration resulted in a time-dependent increase in mitochondrial iron (180 and 160\% 24 and $48 \mathrm{~h}$ after KA injection, respectively) compared with control rats returning to normal values by $7 \mathrm{~d}$. No significant change in chelatable iron was observed in hippocampal cytosolic fractions of rats after KA administration (Fig. 1).

\section{HBED penetrates the $\mathrm{BBB}$ and mitochondria}

Before evaluating its neuroprotective effects, we determined the brain bioavailability of HBED after systemic administration. We measured HBED concentrations in the forebrain and mitochondrial fractions isolated from the hippocampi of rats at different time points after a single injection $(75 \mu \mathrm{mol} / \mathrm{kg}$, s.c). HBED levels were detected $1 \mathrm{~h}$ after administration, peaked at $3 \mathrm{~h}$ and remained stable for at least $6 \mathrm{~h}$. Both tissue and mitochondrial levels remained $\sim 25 \%$ of initial values at the $24 \mathrm{~h}$ time-point after injection (Fig. $2 \mathrm{~A}$ ). These results suggest that $\mathrm{HBED}$ penetrates the $\mathrm{BBB}$ and is accessible to hippocampal mitochondria. HBED has been successfully tested in an animal model of iron-overload, which revealed that multiple injections of $75 \mu \mathrm{mol} / \mathrm{kg}$ was an effective dose for iron elimination with minimal side-effects (Bergeron et al., 


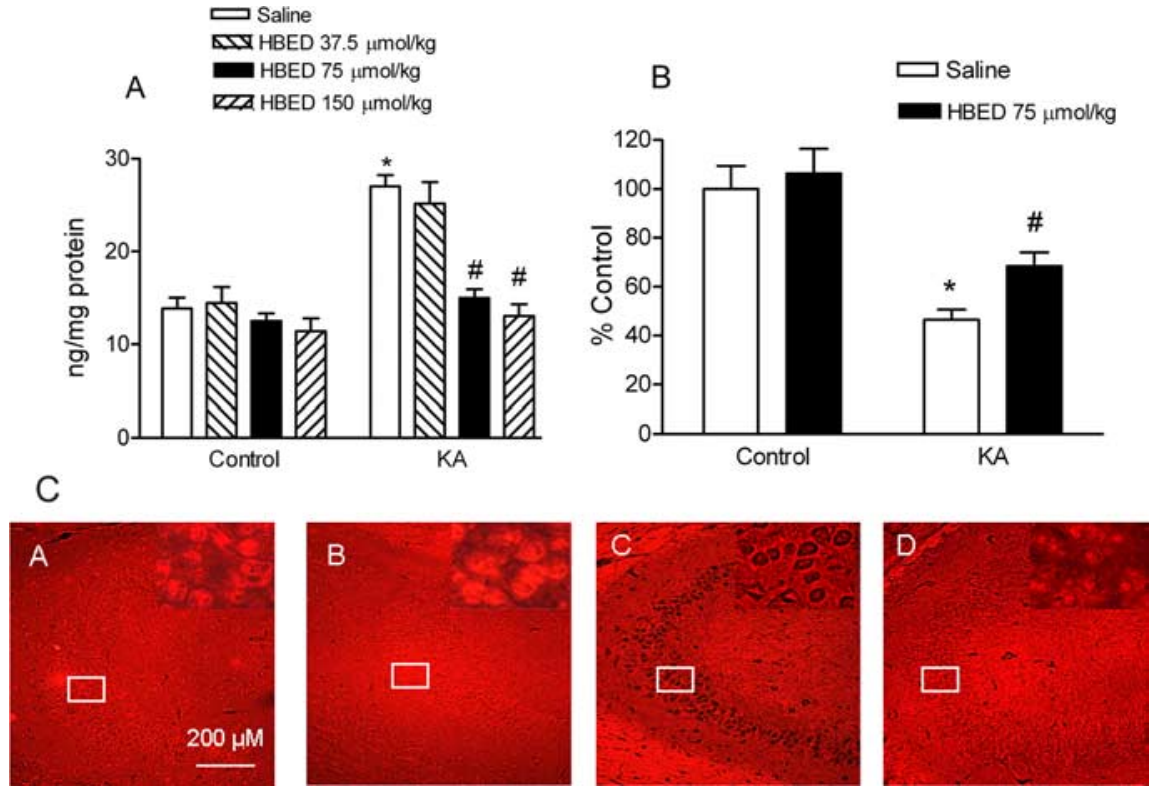

Figure 4. $\boldsymbol{A}, \boldsymbol{B}$, The levels of chelatable iron in mitochondrial fractions measured by the bleomycin method $(\boldsymbol{A})$ or by RPA fluorescence $(\boldsymbol{B})$ in the hippocampus of rats $24 \mathrm{~h}$ after saline, HBED, KA or KA+HBED. Bars represent mean $+\mathrm{SEM},{ }^{*} p<0.01 \mathrm{vs}$ saline controls; ${ }^{*} p<0.05$ vs KA alone; one-way ANOVA, $n=6$ rats per group. $\boldsymbol{C}$, Representative RPA staining images ( $\boldsymbol{A}$, Control; $\boldsymbol{B}, \mathrm{HBED} ; \boldsymbol{C}, \mathrm{KA} ; \boldsymbol{D}, \mathrm{KA}+\mathrm{HBED})$ in (A3 of hippocampus of rats at $24 \mathrm{~h}$ after treatment.

\section{mtDNA damage}
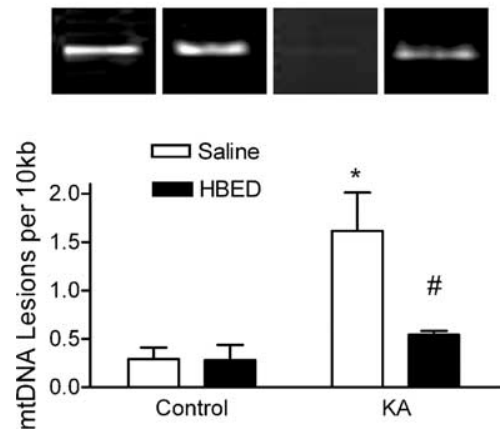

Figure 5. Representative $Q P C R$ images (top) and quantitative analysis (bottom graphs) of mtDNA lesions in hippocampal mitochondria of rats $24 \mathrm{~h}$ after saline, HBED, KA or KA+HBED. The graph represents the number of DNA lesions per $10 \mathrm{~kb}$ in the mitochondrial genome. Bars represent mean + SEM, $^{*} p<0.01$ vs saline controls; ${ }^{\#} p<0.05$ vs KA alone; one-way ANOVA, $n=6-12$ rats per group.

1999, 2002). To confirm the dose of HBED effective in brain iron elimination, the total iron levels in the rat hippocampus were measured with varying doses of HBED. Total iron levels measured by the ferrozine method in the hippocampus were decreased $\sim 30 \%$ after 4 or 5 daily injections with $75 \mu \mathrm{mol} / \mathrm{kg}$. Increasing the injection frequency to 7 resulted in a $\sim 38 \%$ reduction of iron content compared with control animals (Fig. $2 B$ ) but retarded weight gain. Previous studies have demonstrated that a decrease in total iron of $30 \%$ by clioquinol treatment significantly protects against MPTP toxicity (Kaur et al., 2003). This concentration is within the nontoxic range (Yassin et al., 2000) minimally affects most brain biochemical and neurotransmitter metabolism pathways (Youdim and Green, 1978; Youdim et al., 1989). The dose of HBED of $75 \mu \mathrm{mol} / \mathrm{kg}$ was further optimized based on a dose-response study using chelatable iron changes in the mitochondria as an endpoint.

\section{HBED administration does not alter} KA-induced behavioral seizure indices To determine whether the HBED treatment protocol influenced KA-induced behavioral seizures, we evaluated seizure scores, latency to wet dog shakes and duration of SE. No significant differences were observed in behavioral seizure intensity (Fig. 3), seizure latency time $(44.0 \pm 3.4$ and $45.3 \pm 3.2 \mathrm{~min}$ for KA and KA+HBED, respectively; $n=12$ rats per group) and seizure duration $(384.2 \pm 15.4$ and $388.3 \pm 16.1 \mathrm{~min}$ for $\mathrm{KA}$ and $\mathrm{KA}+\mathrm{HBED}$, respectively; $n=12$ rats per group). The percentage of animals that received $\mathrm{KA}$ or $\mathrm{KA}+\mathrm{HBED}$ treatment that did not go on to exhibit a stage 3 or 4 seizure and were thus eliminated from the study was $3.4 \%$ ( 2 of 59 rats) and 3.3\% (2 of 60 rats), respectively. Mortality from KA alone and $\mathrm{KA}+\mathrm{HBED}$ was $5.1 \%$ (3 of 59 rats) and $6.7 \%$ (4 of 60 rats), respectively. No significant differences in mortality rate were observed between KA and KA+HBED groups.

\section{HBED inhibits seizure-induced} chelatable mitochondrial iron changes

Next, we assessed the effect of different doses of HBED on KA-induced increases in mitochondrial chelatable iron $24 \mathrm{~h}$ after KA injection in the four treatment groups. HBED doses of 75 and $150 \mu \mathrm{mol} / \mathrm{kg}$, but not $37.5 \mu \mathrm{mol} / \mathrm{kg}$, for a total 4 injections significantly decreased KA-induced changes in chelatable iron in the hippocampal mitochondrial fractions (Fig. $4 A$ ). Based on the bioavailability parameters and the ability to decrease total as well as chelatable iron, subsequent studies were conducted with the daily HBED dose of $75 \mu \mathrm{mol} / \mathrm{kg}$ for $4 \mathrm{~d}$. The effects of HBED on SE-induced iron chelation were further verified using the RPA fluorescence method. RPA fluorescence quenching has been shown to be a selective indicator of intramitochondrial chelatable iron in both tissue and cells (Petrat et al., 2002a). HBED treatment significantly attenuated the KAinduced increase in mitochondrial chelatable iron detected by RPA fluorescence quenching (Fig. 4B,C). Analysis of RPA fluorescence density revealed that $\mathrm{HBED}$ treatment most prominently attenuated the increase in chelatable iron in the hippocampal CA3 region but also in the CA1 and dentate hilus (data not shown).

\section{HBED protects against SE-induced} mitochondrial dysfunction

To determine whether mitochondrial iron chelation by HBED was sufficient to preserve mitochondrial targets after KA treatment, we analyzed the integrity of mtDNA using a sensitive QPCR assay specific to the mitochondrial genome. KA-induced SE generated an approximate fivefold increase in mtDNA damage in the hippocampus compared with the control group at the $24 \mathrm{~h}$ time point. SE-induced mtDNA damage was significantly attenuated by HBED (Fig. 5).

\section{HBED inhibits SE-induced mitochondrial oxidative stress}

To further determine the deleterious role of mitochondrial iron in SE-induced injury, we measured two additional indices of mitochondrial oxidative stress, the GSH, GSSG and 8-OHdG/2dG ratios. Twenty four hours after KA injection, hippocampal mito- 
chondrial GSH levels were depleted 40\% (Fig. 6A); whereas the corresponding levels of GSSG were significant increased $\sim 240 \%$ compared with controls (Fig. 6 B). The changes in both GSH and GSSG were significantly restored by HBED (Fig. $6 A, B)$. The level of DNA oxidation measured by the ratios of $8-\mathrm{OHdG} / 2 \mathrm{dG}$, was enhanced $>3$-fold by KA-induced SE and attenuated $\sim 50 \%$ by HBED in the hippocampal mitochondrial fractions (Fig. $6 C)$.
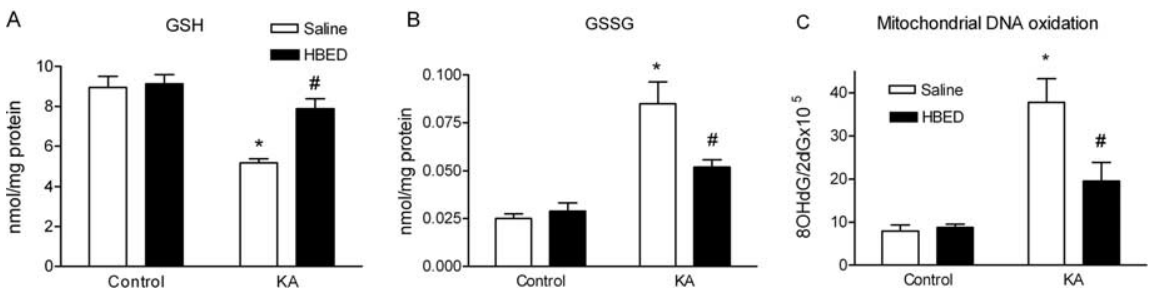

Figure 6. $\boldsymbol{A}-\boldsymbol{C}, \mathrm{GSH}(\boldsymbol{A})$ and GSSG $(\boldsymbol{B}) 80 \mathrm{HdG} / 2 \mathrm{dG}$ ratios, a measure of DNA oxidation $(\boldsymbol{C})$ in hippocampal mitochondrial fractions of rats $24 \mathrm{~h}$ after saline, $\mathrm{HBED}, \mathrm{KA}$ or KA $+\mathrm{HBED}$. Bars represent mean $+\mathrm{SEM},{ }^{*} p<0.01$ vs saline controls; ${ }^{\#} p<0.05$ vs KA alone; one-way ANOVA, $n=6$ rats per group.

\section{HBED protects against SE-induced neuronal degeneration}

Finally, we evaluated the neuroprotective effects of HBED by Fluoro-Jade B fluorescence, a sensitive marker assessing degeneration of neuronal cell bodies, dendrites, axons, or terminals (Hopkins et al., 2000). Previous reports in the literature have demonstrated that Fluoro-Jade B is a more sensitive, reliable and definitive marker of neuronal degeneration than silver staining techniques (Schmued et al., 1997; Schmued and Hopkins, 2000). No significant Fluoro-Jade B staining indicative of degeneration was observed in any brain region of control animals. However, significant staining (degeneration) was observed in the cell bodies and terminals in the hippocampal CA1, CA3 and hilar regions but not granule cell layer of rats injected with KA beginning $\sim 48$ $\mathrm{h}$ (data not shown) and peaking at $\sim 7 \mathrm{~d}$ after injection consistent with previous reports (Schmued et al., 1997; Hopkins et al., 2000). The representative images of Fluoro-Jade B staining of hippocampal CA1, CA3 and hilus of control, KA and KA+HBED groups $7 \mathrm{~d}$ after injection are shown in Figure $7 A$. The percentage of relative fluorescence density quantified by Image $J$ was increased $\sim 190, \sim 240$ and $\sim 265 \%$ in the CA1, CA3 and hilus, respectively in the KA vs control groups. Significant protection of neuronal degeneration was observed in the KA+HBED group compared with KA alone (Fig. $7 B$ ) indicating a neuroprotective effect of HBED.

\section{Discussion}

Three major findings arise from these studies. First, using biochemical and fluorescence approaches, we show that KA-induced SE results in a selective accumulation of mitochondrial chelatable iron, an important free radical catalyst. Second, administration of BBB permeable iron chelator, HBED, ameliorates SE-induced accumulation of chelatable iron, mitochondrial oxidative stress and mtDNA damage. Finally, HBED significantly attenuates SEinduced hippocampal neuronal damage. Collectively, these data suggest a deleterious role of mitochondrial free iron in SEinduced excitotoxicity.

This is the first study to our knowledge demonstrating SEinduced increase in mitochondrial free iron. We used two independent methods, a biochemical method (the bleomycin assay) and a histochemical method (RPA fluorescence assay) to validate the results. Changes in mitochondrial chelatable iron levels using RPA fluorescence quenching have been well defined in cell culture models (Petrat et al., 2002a,b). However, its application to in vivo models has been limited (Rauen et al., 2007). Although RPA fluorescence analysis corroborated the changes in iron observed by the bleomycin assay, it is difficult to rule out the role of dying cells in the fluorescence quenching by RPA in our studies. Consistent with our previous results in which seizure-induced oxidative stress occurred predominantly in the mitochondrial compartment (Liang et al., 2000; Liang and Patel, 2006; Jarrett et al.,
2008), here we show that compared with the cytosol, mitochondria are the main site of chelatable iron accumulation. Furthermore, the time course of mitochondrial iron accumulation closely paralleled the occurrence of oxidative stress we have observed previously. In previous studies, we observed peak changes in mitochondrial oxidative stress indices $\sim 18-24 \mathrm{~h}$ after a single injection of KA (Liang et al., 2000; Liang and Patel, 2006; Patel et al., 2008) and a return to baseline levels $7 \mathrm{~d}$ thereafter (Jarrett et al., 2008).

An important source of mitochondrial iron is the labile ironsulfur center of enzymes such as aconitase. We have previously shown that mitochondrial aconitase inactivation and consequent iron release plays an important role in the neurotoxicity of the parkinsonian toxin, 1-methyl-4-phenyl-1,2,3,6-tetrahydropyridine (MPTP) (Liang and Patel, 2004a). Because the time course of SEinduced mitochondrial aconitase inactivation reported previously from our laboratory (Liang et al., 2000; Jarrett et al., 2008) and iron accumulation match closely, it is tempting to attribute SE-induced iron accumulation at least in part to inactivation of iron-sulfur proteins such as aconitase. Finally, the time course of the iron accumulation observed here preceded overt neuronal death suggesting a pathogenic role of mitochondrial iron in SE-induced neuronal death which was evident $\sim 2 \mathrm{~d}$ after KA and maximal after $7 \mathrm{~d}$.

Mitochondria are recognized to play a crucial role in iron homeostasis (Foury and Talibi, 2001) which includes the synthesis of iron-sulfur clusters (Beinert et al., 1997) and heme (Ryter and Tyrrell, 2000). Histochemical Perl's staining of the hippocampus for iron deposits has revealed increased $\mathrm{Fe}^{3+}$ and $\mathrm{Fe}^{2+}$ deposits $7 \mathrm{~d}$ and 1 month, respectively, after intrahippocampal KA injection (Wang et al., 2002). However, two important differences suggest that iron changes detected in our study most likely represent a pool distinct from that revealed by Perl's staining. First, SE-induced mitochondrial iron accumulation we observed occurred at a much earlier time point (1-2 d) and returned to normal $7 \mathrm{~d}$ after KA injection when the Perl's staining began to emerge. Second, we have previously identified two distinct nonoverlapping pools of iron by the bleomycin method and Perl's staining in the MPTP mouse model (Liang and Patel, 2004a). This suggests that the methods used here detect a different source of iron from that detected by Perl's histochemical staining.

We conducted a comprehensive analysis of the neuroprotective effects of HBED in this study. Measurement of HBED in forebrain tissue and hippocampal mitochondria confirmed its $\mathrm{BBB}$ penetration and access to the mitochondrial compartment. Moreover, both tissue and mitochondrial HBED levels remained $\sim 25 \%$ of the peak values until $24 \mathrm{~h}$ after injection suggesting a relatively long brain half-life. The estimated concentrations of $\sim 10 \mu \mathrm{M}$ in forebrain tissue is in agreement with the concentrations that are neuroprotective against $\mathrm{MPP}^{+}$in cultured cells (Kalivendi et al., 2003). Furthermore, HBED's inability to influence behavioral seizure parameters produced by KA suggests that 
A
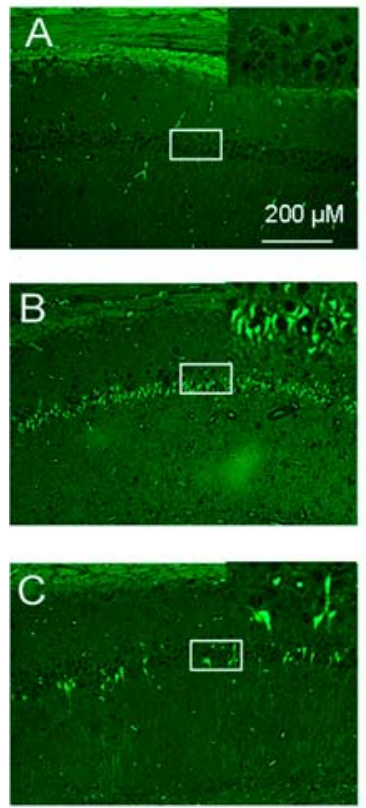

$B$

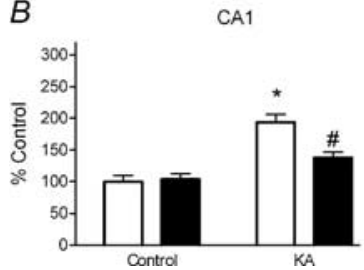

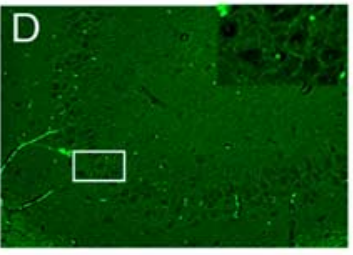
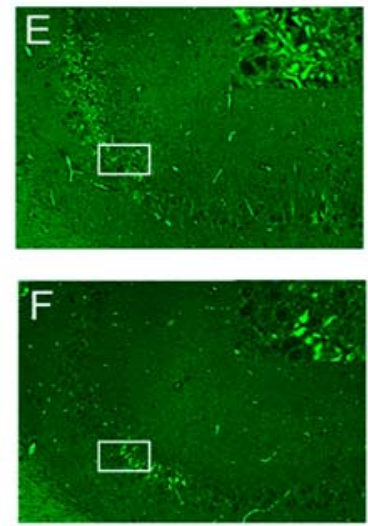

$\mathrm{CA} 3$
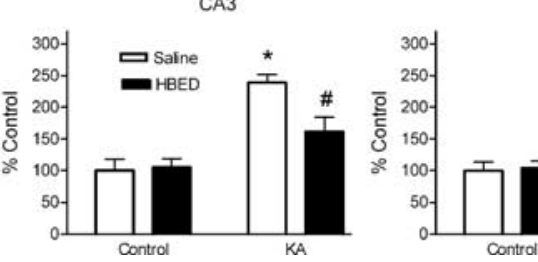

Hilus
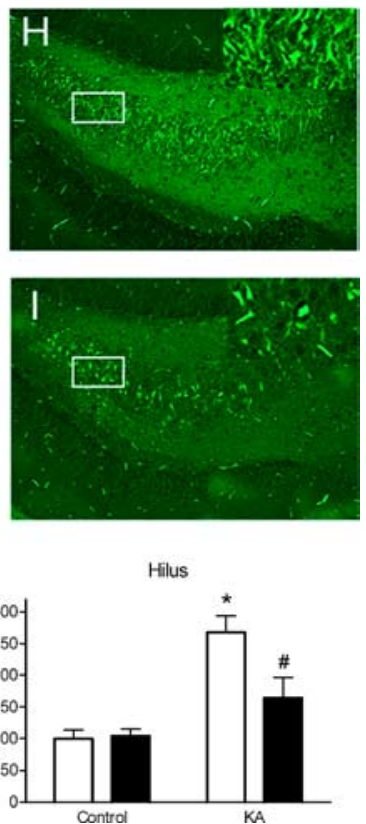

Figure 7. $\boldsymbol{A}$, Representative Fluoro-jade B staining images in the hippocampal regions at $7 \mathrm{~d}$ after KA or KA+HBED.CA1 (A-C) CA3 $(\boldsymbol{D}-\boldsymbol{F})$ and hilus $(\boldsymbol{G}-\boldsymbol{I})$. Control $(\boldsymbol{A}, \boldsymbol{D}, \boldsymbol{G}), \mathrm{KA}(\boldsymbol{B}, \boldsymbol{E}, \boldsymbol{H})$ and $\mathrm{KA}+\mathrm{HBED}(\boldsymbol{C}, \boldsymbol{B}, \boldsymbol{I})$. The insets on the upper right corner of each picture are the enlarged image from the white rectangle. $\boldsymbol{B}$, Quantitative analysis of Fluoro-jade $B$ fluorescence in hippocampal subregions $7 \mathrm{~d}$ after KA or KA + HBED. Bars represent mean $+\mathrm{SEM},{ }^{*} p<0.01$ vs saline controls; ${ }^{*} p<0.05$ vs KA alone; one-way ANOVA, $n=6$ rats per group.

its mechanism of neuroprotection is not related to interference with acute seizure initiation. To effectively evaluate the pharmacological effects of HBED, we determined its effects on mitochondrial iron, its primary target, mitochondrial oxidative stress which may be a cause and/or consequence of the iron, a downstream target of oxidative stress (mtDNA integrity) and neuronal injury. The ability of systemically administered HBED to significantly attenuate mitochondrial iron changes assessed by two independent methods is consistent with and confirms its established role as a mitochondrial iron chelator (Samuni et al., 2001; Kalivendi et al., 2003). The finding that HBED inhibits SEinduced mitochondrial oxidative stress (8-OHdG/2dG and GSH/ GSSG) by $\sim 50 \%$ suggests that the iron released by SE is a catalyst for further free radical production in the mitochondrial compartment and a good target for pharmacological intervention. Protection of an important mitochondrial target (mtDNA) and neuronal integrity suggests that chelation of deleterious mitochondrial iron is a contributing factor to SE-induced mitochondrial dysfunction and neuronal demise. The mitochondrial genome is a particularly vulnerable target for ROS-induced damage and has been implicated as a major factor in the occurrence of epileptic seizures (Liang et al., 2000; Liang and Patel, 2004b, 2006; Patel et al., 2008). The mitochondrial genome encodes components of the electron transport chain and is required for ATP synthesis and maintenance of neuronal excitability. It is plausible that the accumulation of oxidative mtDNA lesions and resultant
mtDNA mutations over time may render the brain more susceptible to subsequent epileptic seizures, especially during the aging process. Thus, HBED-induced sequestration of mitochondrial redox active metal ions may reduce Fenton chemistry, diminish ROS production and maintain genomic stability promoting regular functioning of the mitochondrion and prevention of neuronal excitability. Although in cell-free studies high concentrations of HBED have been shown to possess $\mathrm{H}$-donating antioxidant activity (Samuni et al., 2001), under the conditions of our in vivo study, chelation of mitochondrial iron may be the predominant mechanism of neuroprotection. However, a direct antioxidant action of HBED cannot be ruled out.

Although significant progress has been made in development of new antiepileptic drugs (AEDs) during the last decade, approximately one third of patients develop resistance to the currently available AEDs. Neuroprotective therapies that ameliorate the deleterious consequences of SE may be useful in the management of SE and perhaps even subsequent development of epilepsy. Although therapeutic application of HBED would be better justified if animals had been post-treated with the drug rather than pretreated, it is tempting to speculate that pharmacological chelation of mitochondrial iron may represent an important therapy of the future. Finally, whether elevation of subcellular iron contributes to epileptogenicity in a similar manner as exogenously applied iron salts (Willmore et al., 1978) remains to be determined.

\section{References}

Alam ZI, Daniel SE, Lees AJ, Marsden DC, Jenner P, Halliwell B (1997) A generalised increase in protein carbonyls in the brain in Parkinson's but not incidental Lewy body disease. J Neurochem 69:1326-1329.

Ando K, Ogawa K, Misaki S, Kikugawa K (2002) Increased release of free Fe ions in human erythrocytes during aging in the circulation. Free Radic Res 36:1079-1084.

Andreassen OA, Ferrante RJ, Dedeoglu A, Albers DW, Klivenyi P, Carlson EJ, Epstein CJ, Beal MF (2001) Mice with a partial deficiency of manganese superoxide dismutase show increased vulnerability to the mitochondrial toxins malonate, 3-nitropropionic acid, and MPTP. Exp Neurol 167:189-195.

Arbiser JL, Kraeft SK, van Leeuwen R, Hurwitz SJ, Selig M, Dickersin GR, Flint A, Byers HR, Chen LB (1998) Clioquinol-zinc chelate: a candidate causative agent of subacute myelo-optic neuropathy. Mol Med 4:665-670.

Ayala-Torres S, Chen Y, Svoboda T, Rosenblatt J, Van Houten B (2000) Analysis of gene-specific DNA damage and repair using quantitative polymerase chain reaction. Methods 22:135-147.

Baran H, Lassmann H, Sperk G, Seitelberger F, Hornykiewicz O (1987) Effect of mannitol treatment on brain neurotransmitter markers in kainic acid-induced epilepsy. Neuroscience 21:679-684.

Beard JL, Connor JR, Jones BC (1993) Iron in the brain. Nutr Rev 51:157-170.

Beinert H, Holm RH, Münck E (1997) Iron-sulfur clusters: nature's modular, multipurpose structures. Science 277:653-659.

Ben-Ari Y, Tremblay E, Riche D, Ghilini G, Naquet R (1981) Electrographic, clinical and pathological alterations following systemic administration of 
kainic acid, bicuculline or pentetrazole: metabolic mapping using the deoxyglucose method with special reference to the pathology of epilepsy. Neuroscience 6:1361-1391.

Ben-Shachar D, Eshel G, Finberg JP, Youdim MB (1991) The iron chelator desferrioxamine (Desferal) retards 6-hydroxydopamine-induced degeneration of nigrostriatal dopamine neurons. J Neurochem 56:1441-1444.

Bergeron RJ, Wiegand J, Brittenham GM (1999) HBED: the continuing development of a potential alternative to deferoxamine for iron-chelating therapy. Blood 93:370-375.

Bergeron RJ, Wiegand J, Brittenham GM (2002) HBED ligand: preclinical studies of a potential alternative to deferoxamine for treatment of chronic iron overload and acute iron poisoning. Blood 99:3019-3026.

Brittenham GM (2003) Iron chelators and iron toxicity. Alcohol 30:151-158.

Burkitt MJ, Milne L, Raafat A (2001) A simple, highly sensitive and improved method for the measurement of bleomycin-detectable iron: the 'catalytic iron index' and its value in the assessment of iron status in haemochromatosis. Clin Sci (Lond) 100:239-247.

Cherny RA, Atwood CS, Xilinas ME, Gray DN, Jones WD, McLean CA, Barnham KJ, Volitakis I, Fraser FW, Kim Y, Huang X, Goldstein LE, Moir RD, Lim JT, Beyreuther K, Zheng H, Tanzi RE, Masters CL, Bush AI (2001) Treatment with a copper-zinc chelator markedly and rapidly inhibits beta-amyloid accumulation in Alzheimer's disease transgenic mice. Neuron 30:665-676.

Cock HR (2002) The role of mitochondria and oxidative stress in neuronal damage after brief and prolonged seizures. Prog Brain Res 135:187-196.

Evans PJ, Halliwell B (1994) Measurement of iron and copper in biological systems: bleomycin and copper-phenanthroline assays. Methods Enzymol 233:82-92.

Foury F, Talibi D (2001) Mitochondrial control of iron homeostasis. A genome wide analysis of gene expression in a yeast frataxin-deficient strain. J Biol Chem 276:7762-7768.

Glickstein H, El RB, Shvartsman M, Cabantchik ZI (2005) Intracellular labile iron pools as direct targets of iron chelators: a fluorescence study of chelator action in living cells. Blood 106:3242-3250.

Halliwell B, Gutteridge JM (1990) Role of free radicals and catalytic metal ions in human disease: an overview. Methods Enzymol 186:1-85.

Hammond EJ, Ramsay RE, Villarreal HJ, Wilder BJ (1980) Effects of intracortical injection of blood and blood components on the electrocorticogram. Epilepsia 21:3-14.

Henshall DC, Simon RP (2005) Epilepsy and apoptosis pathways. J Cereb Blood Flow Metab 25:1557-1572.

Hill JM, Switzer RC 3rd (1984) The regional distribution and cellular localization of iron in the rat brain. Neuroscience 11:595-603.

Hopkins KJ, Wang G, Schmued LC (2000) Temporal progression of kainic acid induced neuronal and myelin degeneration in the rat forebrain. Brain Res 864:69-80.

Jarrett SG, Liang LP, Hellier JL, Staley KJ, Patel M (2008) Mitochondrial DNA damage and impaired base excision repair during epileptogenesis. Neurobiol Dis 30:130-138.

Kalivendi SV, Kotamraju S, Cunningham S, Shang T, Hillard CJ, Kalyanaraman B (2003) 1-Methyl-4-phenylpyridinium (MPP+)-induced apoptosis and mitochondrial oxidant generation: role of transferrin-receptor-dependent iron and hydrogen peroxide. Biochem J 371:151-164.

Kasai H, Crain PF, Kuchino Y, Nishimura S, Ootsuyama A, Tanooka H (1986) Formation of 8-hydroxyguanine moiety in cellular DNA by agents producing oxygen radicals and evidence for its repair. Carcinogenesis 7:1849-1851.

Kaur D, Yantiri F, Rajagopalan S, Kumar J, Mo JQ, Boonplueang R, Viswanath V, Jacobs R, Yang L, Beal MF, DiMonte D, Volitaskis I, Ellerby L, Cherny RA, Bush AI, Andersen JK (2003) Genetic or pharmacological iron chelation prevents MPTP-induced neurotoxicity in vivo: a novel therapy for Parkinson's disease. Neuron 37:899-909.

Kirk EA, Heinecke JW, LeBoeuf RC (2001) Iron overload diminishes atherosclerosis in apoE-deficient mice. J Clin Invest 107:1545-1553.

Kudin AP, Kudina TA, Seyfried J, Vielhaber S, Beck H, Elger CE, Kunz WS (2002) Seizure-dependent modulation of mitochondrial oxidative phosphorylation in rat hippocampus. Eur J Neurosci 15:1105-1114.

Lakritz J, Plopper CG, Buckpitt AR (1997) Validated high-performance liquid chromatography-electrochemical method for determination of glutathione and glutathione disulfide in small tissue samples. Anal Biochem 247:63-68.

Liang LP, Patel M (2004a) Iron-sulfur enzyme mediated mitochondrial su- peroxide toxicity in experimental Parkinson's disease. J Neurochem 90:1076-1084.

Liang LP, Patel M (2004b) Mitochondrial oxidative stress and increased seizure susceptibility in Sod2 $(-/+)$ mice. Free Radic Biol Med 36:542-554.

Liang LP, Patel M (2006) Seizure-induced changes in mitochondrial redox status. Free Radic Biol Med 40:316-322.

Liang LP, Ho YS, Patel M (2000) Mitochondrial superoxide production in kainate-induced hippocampal damage. Neuroscience 101:563-570.

May ME, Fish WW (1978) The UV and visible spectral properties of ferritin. Arch Biochem Biophys 190:720-725.

Patel M (2004) Mitochondrial dysfunction and oxidative stress: cause and consequence of epileptic seizures. Free Radic Biol Med 37:1951-1962.

Patel M, Liang LP, Hou H, Williams BB, Kmiec M, Swartz HM, Fessel JP, Roberts LJ 2nd (2008) Seizure-induced formation of isofurans: novel products of lipid peroxidation whose formation is positively modulated by oxygen tension. J Neurochem 104:264-270.

Petrat F, de Groot H, Rauen U (2001) Subcellular distribution of chelatable iron: a laser scanning microscopic study in isolated hepatocytes and liver endothelial cells. Biochem J 356:61-69.

Petrat F, de Groot H, Sustmann R, Rauen U (2002a) The chelatable iron pool in living cells: a methodically defined quantity. Biol Chem 383:489-502.

Petrat F, Weisheit D, Lensen M, de Groot H, Sustmann R, Rauen U (2002b) Selective determination of mitochondrial chelatable iron in viable cells with a new fluorescent sensor. Biochem J 362:137-147.

Rauen U, Springer A, Weisheit D, Petrat F, Korth HG, de Groot H, Sustmann R (2007) Assessment of chelatable mitochondrial iron by using mitochondrion-selective fluorescent iron indicators with different ironbinding affinities. Chembiochem 8:341-352.

Riemer J, Hoepken HH, Czerwinska H, Robinson SR, Dringen R (2004) Colorimetric ferrozine-based assay for the quantitation of iron in cultured cells. Anal Biochem 331:370-375.

Ryter SW, Tyrrell RM (2000) The heme synthesis and degradation pathways: role in oxidant sensitivity. Heme oxygenase has both pro- and antioxidant properties. Free Radic Biol Med 28:289-309.

Samuni AM, Afeworki M, Stein W, Yordanov AT, DeGraff W, Krishna MC, Mitchell JB, Brechbiel MW (2001) Multifunctional antioxidant activity of HBED iron chelator. Free Radic Biol Med 30:170-177.

Schmued LC, Hopkins KJ (2000) Fluoro-Jade B: a high affinity fluorescent marker for the localization of neuronal degeneration. Brain Res 874:123-130.

Schmued LC, Albertson C, Slikker W Jr (1997) Fluoro-Jade: a novel fluorochrome for the sensitive and reliable histochemical localization of neuronal degeneration. Brain Res 751:37-46.

Shigenaga MK, Park JW, Cundy KC, Gimeno CJ, Ames BN (1990) In vivo DNA damage: measurement of 8-hydroxy-2' deoxyguanosine in DNA and urine by high-performance liquid chromatography with electrochemical detection. Methods Enzymol 186:521-530.

Shoham S, Youdim MB (2004) Nutritional iron deprivation attenuates kainate-induced neurotoxicity in rats: implications for involvement of iron in neurodegeneration. Ann N Y Acad Sci 1012:94-114.

Sohal RS, Wennberg-Kirch E, Jaiswal K, Kwong LK, Forster MJ (1999) Effect of age and caloric restriction on bleomycin-chelatable and nonheme iron in different tissues of C57BL/6 mice. Free Radic Biol Med 27:287-293.

Wang XS, Ong WY, Connor JR (2002) Increase in ferric and ferrous iron in the rat hippocampus with time after kainate-induced excitotoxic injury. Exp Brain Res 143:137-148.

Willmore LJ, Sypert GW, Munson JB (1978) Recurrent seizures induced by cortical iron injection: a model of posttraumatic epilepsy. Ann Neurol 4:329-336.

Yakes FM, Van Houten B (1997) Mitochondrial DNA damage is more extensive and persists longer than nuclear DNA damage in human cells following oxidative stress. Proc Natl Acad Sci U S A 94:514-519.

Yassin MS, Ekblom J, Xilinas M, Gottfries CG, Oreland L (2000) Changes in uptake of vitamin $\mathrm{B}(12)$ and trace metals in brains of mice treated with clioquinol. J Neurol Sci 173:40-44.

Youdim MB, Green AR (1978) Iron deficiency and neurotransmitter synthesis and function. Proc Nutr Soc 37:173-179.

Youdim MB, Ben-Shachar D, Yehuda S (1989) Putative biological mechanisms of the effect of iron deficiency on brain biochemistry and behavior. Am J Clin Nutr 50:607-615, discussion 615-617.

Zecca L, Youdim MB, Riederer P, Connor JR, Crichton RR (2004) Iron, brain ageing and neurodegenerative disorders. Nat Rev Neurosci 5:863-873. 